\title{
Enamel cracks evaluation -A method to predict tooth surface damage during the debonding
}

\author{
Irma DUMBRYTE¹, Tomas JONAVICIUS ${ }^{2}$, Laura LINKEVICIENE³ ${ }^{3}$ Tomas LINKEVICIUS ${ }^{3}$, Vytaute PECIULIENE ${ }^{3}$ \\ and Mangirdas MALINAUSKAS ${ }^{2}$
}

\author{
${ }^{1}$ Vilnius Research Group, Polocko 21/Zvirgzdyno 1, Vilnius LT-01205, Lithuania \\ ${ }^{2}$ Department of Quantum Electronics, Physics Faculty, Vilnius University, Sauletekio 9, Vilnius LT-10222, Lithuania \\ ${ }^{3}$ Institute of Odontology, Faculty of Medicine, Vilnius University, Zalgirio 115, Vilnius LT-08217, Lithuania \\ Corresponding author, Irma DUMBRYTE; E-mail: i.dumbryte@gmail.com
}

\begin{abstract}
The objective of this in vitro study was to evaluate the effect of the enamel cracks on the tooth damage during the debonding. Measurements of the cracks characteristics (visibility, direction, length, and location) were performed utilizing a scanning electron microscopy (SEM) technique and mathematically derived formulas $(\mathrm{x}=\mathrm{h} / 30, \mathrm{l}=\mathrm{n} * \mathrm{x})$ before and following the removal of mechanically retained metal and ceramic brackets. The likelihood of having greater extent enamel defects was higher for the teeth with pronounced cracks (odds vatios, OR=3.728), increased when the crack was located in more than one zone of the tooth $(\mathrm{OR}=1.998)$, and the inclination did not exceed 30-45 $(\mathrm{OR}=0.505)$. Using ceramic brackets the risk of greater amount tooth structure defects raised 1.45 times $(\mathrm{OR}=1.450)$. Enamel crack showing all these characteristics at the beginning of the orthodontic treatment and the use of ceramic brackets might predispose to higher risk of greater extent tooth surface damage after the debonding by $20.4 \%$.
\end{abstract}

Keywords: Bracket, Damage, Crack, Scanning electron microscope, Structure

\section{INTRODUCTION}

The question how to avoid enamel surface damage during treatment with fixed appliances has become important together with the introduction of the first brackets systems. Since then, attempts have been made to provide patients with the orthodontic therapy that causes minimal undesirable changes in the enamel structure. Various enamel surface modification techniques, the protocols for teeth etching and bonding have been described in respect of the enamel damage following the removal of brackets ${ }^{1-4)}$. Even more attention has been paid to the effect of the debonding procedure on the changes in the tooth structure ${ }^{5-8}$. However, little is known about the role of the cracks itself to the development of greater extent enamel surface defects after the removal of brackets.

The formation of the enamel cracks has generally been attributed to the abnormalities in the maturation process, occlusal forces (e.g. generated during dental attrition and abrasion), traumatic injuries (such as extraction procedure), temperature variations, and restorative processes ${ }^{9,10)}$. According to previously performed studies, micro-cracks can be noticed after the debonding at the end of the orthodontic treatment ${ }^{11,12)}$. It is presented in the existing literature that human enamel has a complex microstructure that can contribute to fracture toughness and crack growth resistance under various mechanical load conditions ${ }^{13)}$. Previous investigations have demonstrated the behavior of the enamel micro-cracks during the debonding and showed

Color figures can be viewed in the online issue, which is available at J-STAGE.

Received Mar 16, 2015: Accepted Jun 10, 2015

doi:10.4012/dmj.2015-085 JOI JST.JSTAGE/dmj/2015-085 results about the changes in the direction, length, number, and width of the cracks after the removal of metal or ceramic brackets ${ }^{1,7,12)}$. Still, it has not been looked for the relationship between the specific microcracks characteristics at the beginning of the orthodontic treatment and the enamel damage during the debonding procedure.

Thus, the objective of this in vitro study was to evaluate the effect of the qualitative cracks characteristics (visibility, direction, length, and location) on the enamel surface damage during the removal of brackets. Additional aim was to observe if the predictions about the irreversible changes in the tooth structure following the debonding could be made from a set of the cracks characteristics and type of the bracket used at the beginning of the treatment.

The null hypothesis was that the qualitative microcracks characteristics (visibility, direction, length, and location) do not have any effect on the enamel damage formation during the debonding.

\section{MATERIALS AND METHODS}

Teeth selection and enamel surface evaluation

The teeth were prepared in accordance with the guidelines of the International Organization for Standardization ${ }^{14)}$.

Sixty extracted maxillary premolars were included in the final study sample. The teeth had been extracted for orthodontic reasons and were used with the patients' informed consent. The selection criteria for the teeth are given in Table 1. All the teeth were decontaminated in $0.5 \%$ chloramine $\mathrm{T}$ solution and stored in distilled 
water that was changed weekly before preparation and testing.

Study protocol is presented in Fig. 1. The buccal enamel surfaces of all the teeth included in the investigation were evaluated utilizing a scanning electron microscopy (SEM, Hitachi TM-1000, Japan) as described previously ${ }^{12}$. The SEM was operated at 15 $\mathrm{kV}$, at less than $5 \times 10^{-2} \mathrm{~Pa}$ (electron gun vacuum) and at approximately 30-50 $\mathrm{Pa}$ (specimen chamber vacuum). The teeth were not coated with a conductive layer prior to SEM examination. The initial evaluation of microcracks was performed at $\times 50-100$.

The examination of the buccal enamel surface using the SEM technique is shown in Fig. 2. The SEM micrographs of the buccal enamel surfaces of all the teeth having micro-cracks were taken. Montages (stitching together of multiple images) of the SEM micrographs were made to reconstruct images of some larger crowns. From digital SEM micrographs, vertical height (h) of every tooth's crown was evaluated. For detailed mapping of enamel cracks, the buccal surface was divided in three zones of equal height: 1st zone - cervical third, 2nd zone -middle third, 3rd zone - occlusal third ${ }^{1,5,12)}$. With a help of a constructed ruler, every zone was divided in 10 measurement areas (MA), and a total of 30 MA of every tooth were obtained. With a help of a mathematically derived formula, a measurement step ( $x$, the distance between two MA) was quantified (Fig. 2). Despite the existing number of enamel cracks, only the longest one was chosen and evaluated in detail. Qualitative micro-cracks characteristics were analyzed: visibility, direction, length, and location.

The direction of enamel crack was classified as: vertical $\left(0-30^{\circ}\right.$ to the long axis of the crown), oblique (31-45 to the long axis of the crown), and horizontal (46-90 to the long axis of the crown). The length of the longest micro-crack was quantified with the help of a formula (Fig. 2). The location was specified as occlusal, middle and cervical third of the buccal tooth surface. On the basis of the visual examination of the buccal enamel surface, micro-cracks were categorized to weak (not apparent under normal room illumination but can be visualized with use of the SEM) and pronounced ones (visible with a naked eye under normal room illumination ${ }^{5,15)}$ ).

Following the initial examination with the SEM, the teeth having enamel cracks were randomly divided into two groups of 30: group 1, the teeth were bonded with metal brackets, group 2, ceramic brackets were used.

Control group was not included in the study because there was no statistical comparability.

\section{Bonding procedure}

In group 1, mechanically retained maxillary premolar metal brackets (Discovery, Dentaurum, Germany), with 0.022 -inch slots were used. The average surface area of

Table 1 Teeth selection criteria

Primary teeth selection criteria

- Intact buccal enamel surfaces with no white spots and signs of dental fluorosis.

- No pre-treatment with chemical agents (such as hydrogen peroxide).

- No previous endodontic, orthodontic, and restorative treatment.

Secondary teeth selection criteria

- Enamel cracks on the buccal enamel surface.

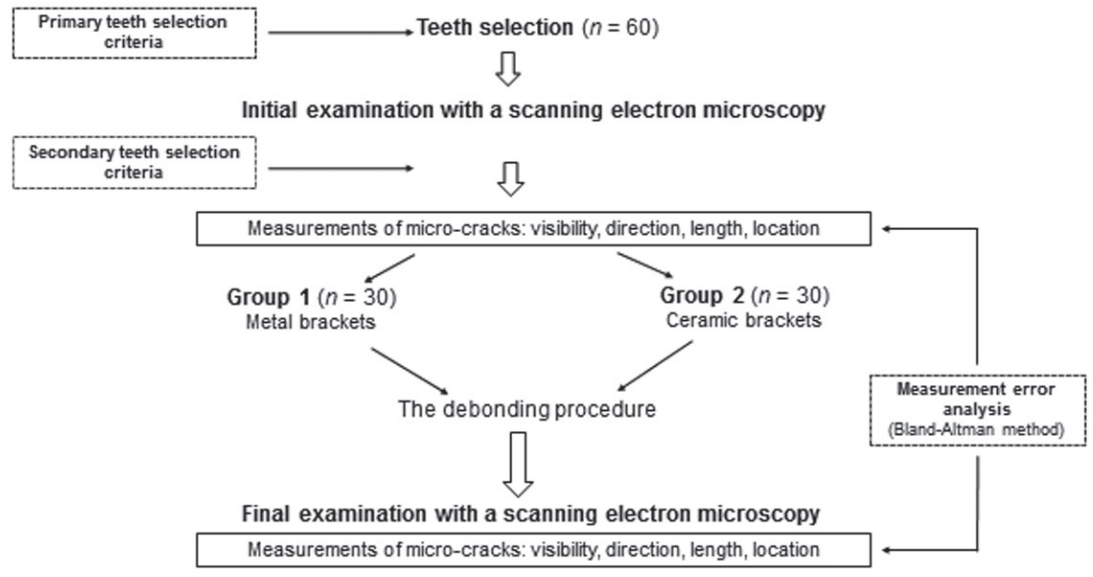

Fig. 1 A graphic representation of the study protocol. 


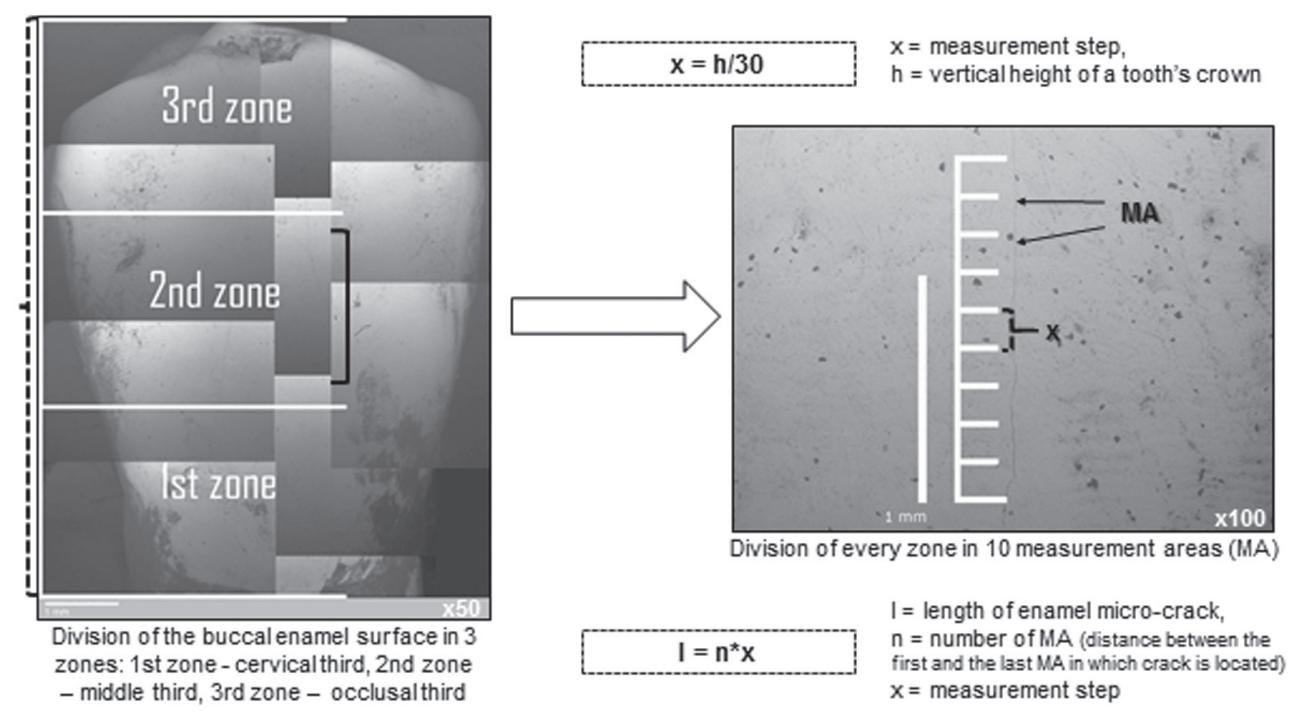

Fig. 2 An examination of the buccal enamel surface using the SEM technique and mathematically derived formulas.

the bracket base was measured and recorded as $11.9 \mathrm{~mm}^{2}$. The teeth were prepared according to this protocol: etching with $34.5 \%$ phosphoric acid gel (Vococid, Voco, Germany) for $30 \mathrm{~s}$, rinsing with water for $20 \mathrm{~s}$, drying with air for $10 \mathrm{~s}$, applying the primer (Contex Primer, Dentaurum) and curing it with light for $10 \mathrm{~s}$. The bonding base of metal bracket was applied with a similar amount of resin adhesive (Transbond XT, 3M Unitek, USA), the bracket was firmly positioned on the enamel surface with the help of bracket holding tweezer, and the excess adhesive was removed from around the base of the bracket with an explorer, this way ensuring uniform resin adhesive thickness. The light-cure adhesive was polymerized for $20 \mathrm{~s}$ (10 s from each proximal side) using a halogen light (Mini L.E.D., Satelec).

In group 2, maxillary premolar ceramic brackets (Clarity, 3M Unitek) with mechanical bases and 0.022inch metal slots were used. The average base area was $11.83 \mathrm{~mm}^{2}$. All the teeth were bonded according to the same procedure that was described for group 1 .

After bonding, all the teeth were placed in distilled water at $37^{\circ} \mathrm{C}$ and stored for $24 \mathrm{~h}$ prior to testing ${ }^{14)}$.

\section{Debonding procedure}

Debonding of metal brackets was carried out with conventional Utility/Weingart (Dentaurum) pliers by hand. The mesio-distal edges of the bracket wings were squeezed gently until the bracket was removed ${ }^{5)}$.

Ceramic brackets were removed with the help of Debonding instrument (3M Unitek) on the basis of the manufacturers' recommendations. The pliers were placed against the mesial and distal sides of the bracket and were positioned symmetrically against the labial surface of the bracket to optimize contact surface area. The instrument was gently squeezed until the bracket collapsed, then was gently rocked in the mesial-distal direction until the bracket became completely separated from the enamel. All metal and ceramic brackets were removed by the operator having seven year experience in clinical orthodontic practice and who has already participated in similar studies.

All visible residual adhesive was carefully removed from the surface of the teeth using a slow-speed handpiece and a carbide-finishing bur, under normal clinical conditions ${ }^{16)}$. Light movements of the bur were used in order not to scratch the enamel. Water cooling was not employed when the last remnants were removed, and polishing instruments were not utilized. Following the debonding, the enamel surface was reevaluated with the SEM as described previously.

Measurement error was tested and parameters were presented in the earlier published study ${ }^{12)}$.

\section{Statistical analysis}

Statistical analysis was carried out using the Statistical Package SPSS 17.0. Binary logistic regression was applied to predict the extent of tooth damage following the removal of brackets from a set of the cracks characteristics and type of the bracket used. Calculated length values of the cracks represented enamel surface defects after the debonding. Dependent variable was length of the crack, which was converted from continuous scale to nominal, using variance analysis of continuous values [(0.24 thru 2.16=1) (2.17 thru 9.16=2); 1-small/low, 2-large/high]. Four independent variables (direction, location, visibility, and bracket type) were included in the model. To visualize their impact on the extent of tooth damage following the debonding, mosaic charts were utilized ${ }^{17)}$. Statistical significance was based on $p$ values and $95 \%$ confidence interval. 


\section{RESULTS}

Impact of four different factors (direction, location, visibility of the micro-crack, and bracket type) on the extent of enamel surface damage during the removal of brackets is presented in Table 2 .

The location of the enamel micro-crack had significant effect on the extent of tooth damage during the debonding procedure $(\mathrm{OR}=1.998 ; p<0.05)$. The odds of greater undesirable changes in the enamel microstructure following the removal of brackets were higher for the micro-cracks located in more than one zone of the tooth. $34.8-50.0 \%$ of the cracks occupying two neighboring zones and $100 \%$ of the cracks that extended through the whole buccal tooth surface predisposed to the increase in the risk of greater amount tooth damage after the debonding (Table 3). For an additional millimeter in the distance (i.e., length of the micro-crack), the odds of

Table 2 Impact of four different factors on the extent of enamel surface damage during the debonding (dependent variable -length value)

\begin{tabular}{|c|c|c|c|c|}
\hline \multirow{2}{*}{ Independent variables } & \multirow{2}{*}{$p$} & \multirow{2}{*}{$\mathrm{OR}^{*}$} & 95\% C.I. for OR & 95\% C.I. for OR \\
\hline & & & lower & upper \\
\hline Direction of the crack** & 0.170 & 0.505 & 0.190 & 1.340 \\
\hline Location of the $\operatorname{crack}^{* * *}$ & 0.002 & 1.998 & 1.285 & 3.105 \\
\hline Visibility of the $\operatorname{crack}^{* * *}$ & 0.005 & 3.728 & 1.489 & 9.335 \\
\hline Bracket type $e^{* * * *}$ & 0.385 & 1.450 & 0.627 & 3.355 \\
\hline
\end{tabular}

* OR values: 1=independent variable has no impact; <1=lowers the risk; >1=increases the risk of greater extent enamel surface damage during the debonding.

${ }^{* *}$ OR $<1$ and its' Upper 95\% C.I. value $>1$, variable statistically insignificantly lowers the risk of greater extent tooth damage formation.

$* * *$ OR $>1$ and its' Lower 95\% C.I. value $>1$, variable statistically significantly increases the risk of greater amount enamel surface defects.

$* * * *$ OR $>1$ and its' Lower 95\% C.I. value $<1$, variable statistically insignificantly increases the risk of greater extent enamel damage formation during the debonding.

$p$ Values, odds ratios (OR) and 95\% confidence intervals (C.I.) from binary logistic modeling.

Table 3 Relationship between the extent of enamel damage after the debonding and location of the micro-crack

\begin{tabular}{llr}
\multicolumn{1}{c}{ Location of the crack* } & \multicolumn{2}{c}{ Extent of damage (\%) } \\
\cline { 2 - 3 } Occlusal third or occlusal and middle third & low & 50.0 \\
Middle third & 50.0 & 71.4 \\
Middle and cervical third or cervical third & 65.2 & 34.8 \\
Occlusal, middle, and cervical third & - & 100.0 \\
\hline
\end{tabular}

* Just few enamel micro-cracks were located in one zone, except for the middle third, and most of them were observed in two or three zones. Thus, during the statistical analysis four groups were composed to represent common location alternatives for the precise statistical evaluation.

Table 4 Relationship between the extent of enamel damage after the debonding and direction of the micro-crack

\begin{tabular}{ccc}
\hline & \multicolumn{2}{c}{ Extent of damage (\%) } \\
\cline { 2 - 3 } Direction of the crack & low & 52.1 \\
Vertical & 47.9 & 42.9 \\
Oblique & 57.1 & - \\
Horizontal & 100.0 & - \\
\hline
\end{tabular}




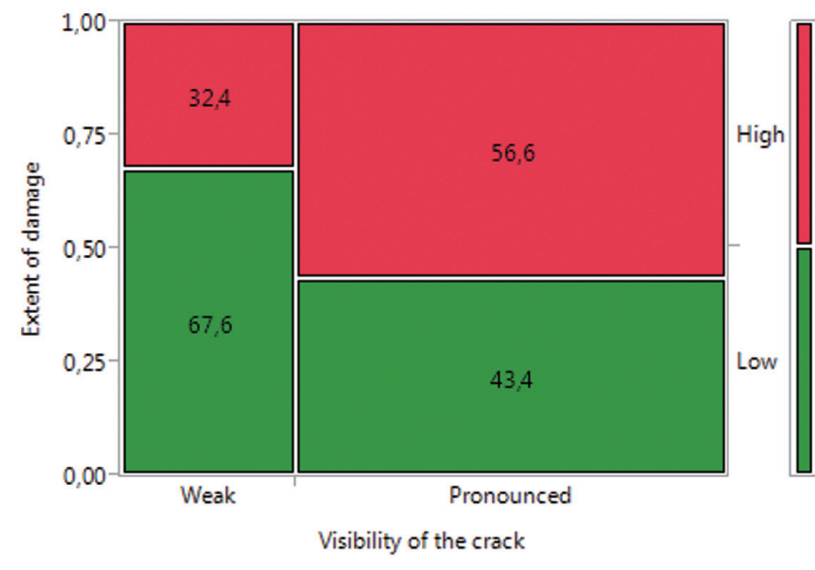

Fig. 3 A mosaic chart.

The relationship between the extent of enamel damage after the debonding and visibility of the micro-crack.

greater enamel damage were higher by a factor of 1.998 (99.8\%), that is, statistically significantly almost doubled the risk of greater extent tooth structure defects.

The direction of the micro-crack did not lead to significant changes in the enamel structure after the debonding $(\mathrm{OR}=0.505 ; p>0.05)$. A more detailed evaluation showed that $52.1 \%$ of vertical and $42.9 \%$ of oblique cracks predisposed to higher risk of greater extent tooth surface damage following the removal of brackets (Table 4). For 1\% degree more inclined enamel micro-cracks, the odds of greater undesirable changes in the enamel structure decreased by a factor of 0.505 (49.5\%).

Visibility of the crack had a huge and statistically significant effect on the amount of tooth damage during the debonding $(\mathrm{OR}=3.728 ; p<0.05)$. The odds of greater enamel damage were lower for weak micro-cracks. $32.4 \%$ of weak cracks and $56.6 \%$ of pronounced ones led to the increase in the risk of greater extent tooth surface defects formation (Fig. 3). For more pronounced cracks, the odds of the undesirable changes in the enamel structure were higher by a factor of 3.728 , thus, almost four times increased the risk of greater amount tooth damage after the removal of brackets.

Bracket type showed having an impact on the extent of enamel structure defects following the debonding $(\mathrm{OR}=1.450 ; p>0.05) .40 .4 \%$ of the teeth bonded with metal and $58.3 \%$ with ceramic brackets predisposed to the increase in the risk of greater amount enamel surface damage after the removal procedure (Fig. 4). Using ceramic brackets, the odds of having greater extent tooth structure defects increased by a factor of $1.450(45 \%)$.

The coefficient of determination (Nagelkerke $\mathrm{R}^{2}=0.204$ ) presented that all four independent variables had an impact on the extent of enamel damage after the debonding by $\sim 20.4 \%$, the null hypothesis was rejected.

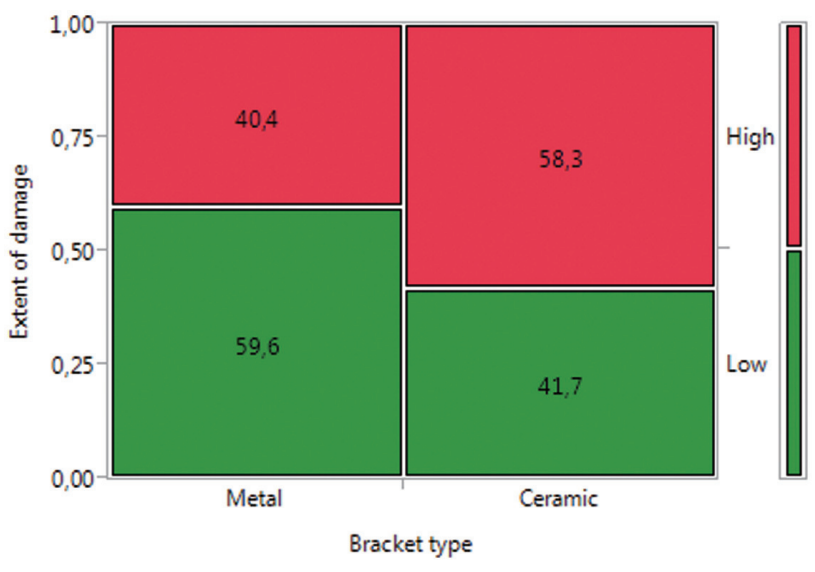

Fig. 4 A mosaic chart.

The relationship between the extent of enamel damage after the debonding and bracket type.

\section{DISCUSSION}

The study aimed at the evaluation of the effect of the micro-cracks on the enamel damage during the removal of brackets. Additional objective was to observe if the predictions about the irreversible changes in the tooth structure following the debonding could be made from a set of the cracks characteristics and type of the bracket used at the beginning of the treatment.

Location (occlusal, middle and cervical third) and visibility (weak and pronounced) of the crack were two independent variables that statistically significantly increased the risk of greater amount enamel surface defects after the removal of brackets. Analysis of the obtained results showed that $34.8-50.0 \%$ of the microcracks located in two neighboring zones (occlusal and middle third or middle and cervical third) or extending through the whole buccal tooth surface (occlusal, middle and cervical third) could double the possibility of the undesirable changes in the enamel structure following the debonding. It seems that occlusal and cervical parts of the tooth surface, but not the middle one, are more prone to damage during the removal of brackets. According to the existing literature, after the debonding the greatest irreversible changes in the tooth structure occur in the cervical third of the buccal tooth surface, then followed by occlusal third ${ }^{2,12,15,18)}$. The higher risk to develop damage in these zones might be related with the mechanical properties (hardness, elastic modulus, and fracture toughness) of human enamel ${ }^{19,20)}$.

Visibility of the enamel micro-crack was the characteristic that predisposed to the highest risk of greater extent enamel damage formation following the removal of brackets. $56.6 \%$ of pronounced and $32.4 \%$ of weak cracks led to the increase in the possibility of greater amount tooth surface defects. Despite the fact that nowadays more and more patients notice enamel cracks and wonder about their effect on the tooth 
structure, the characteristics of the pronounced microcracks are poorly described in the literature. Just few facts are known about the changes in the number after the debonding ${ }^{7}$. The results of our study revealed that for more pronounced cracks, the odds of the undesirable changes in the enamel structure were higher by a factor of 3.728, thus, almost four times increased the risk of greater amount tooth structure defects after the removal of brackets. So the effect of these cracks on the enamel damage following the debonding should not be neglected and further studies are necessary for more detailed evaluation of the pronounced micro-cracks characteristics.

Examination of the direction parameter showed that this independent variable did not lead to significant changes in the enamel structure after the debonding. A more detailed analysis revealed that $52.1 \%$ of vertical and $42.9 \%$ of oblique cracks predisposed to higher risk of greater extent tooth damage following the removal of brackets. Previous investigations have demonstrated that horizontal micro-cracks possessed high risk of underlying pathology ${ }^{21)}$. Thus, it was surprising to see that in our study horizontal cracks were related with low possibility of higher amount enamel defects. The difference in the results can be explained by several reasons. First of all, during the evaluation of the teeth with the SEM, we have found only two horizontal microcracks that were included in the further investigation; sample size was not enough to examine the behavior of horizontal cracks. Secondly, in other cases, where several cracks of different directions were observed, horizontal cracks were left aside because they were not the longest ones. Vertical micro-cracks showed the highest length values.

During the evaluation of the effect of the bracket type on the extent of enamel damage following the debonding, it was noticed that $40.4 \%$ of the teeth bonded with metal and $58.3 \%$ with ceramic brackets predisposed to higher risk of greater amount tooth structure defects after the removal procedure. Previous studies analyzing the impact of the debonding on the enamel have not come to one common conclusion yet. Thus, the results vary from no enamel damage after the removal both types of brackets, to $20.0-40.0 \%$ of the teeth with new enamel cracks following the debonding (25.0-40.0\% after the removal of metal and 20.0$35.0 \%$ - ceramic brackets), and finally, to equal risk of tooth structure defects when debonding both types of brackets $^{4,5,11,12,22)}$. However, according to our obtained results, using ceramic brackets, the odds of having greater extent tooth structure defects increased by a factor of $1.450(45 \%)$.

\section{CONCLUSION}

Enamel micro-cracks characteristics (visibility, location, and direction) and bracket type predisposed to higher risk of greater amount tooth surface damage following the debonding by $20.4 \%$. This shows that many factors are related with the formation of the enamel structure defects during the orthodontic therapy with fixed appliances and existing before treatment micro-cracks are one of them. Although the exact role of other possible factors is not known, the results of our study might help getting closer to making predictions of the irreversible changes in the tooth structure at the beginning of the orthodontic treatment.

\section{REFERENCES}

1) Shahabi M, Heravi F, Mokhber N, Karamad R, Bishara SE. Effects on shear bond strength and the enamel surface with an enamel bonding agent. Am J Orthod Dentofacial Orthop 2010; $137:$ 375-378.

2) Tecco S, Tetè S, D'Attilio M, Festa F. Enamel surface after debracketing of orthodontic brackets bonded with flowable orthodontic composite. A comparison with a traditional orthodontic composite resin. Minerva Stomatol 2008; 57: 8194.

3) Lamper T, Ilie N, Huth KC, Rudzki I, Wichelhaus A, Paschos E. Self-etch adhesives for the bonding of orthodontic brackets: faster, stronger, safer? Clin Oral Investig 2014; 18: 313-319.

4) Elekdag-Turk S, Isci D, Ozkalayci N, Turk T. Debonding characteristics of a polymer mesh base ceramic bracket bonded with two different conditioning methods. Eur J Orthod 2009; 31: 84-89.

5) Bishara SE, Ostby AW, Laffoon J, Warren JJ. Enamel cracks and ceramic brackets failure during debonding in vitro. Angle Orthod 2008; 78: 1078-1083.

6) Heravi F, Rashed R, Raziee L. The effects of bracket removal on enamel. Aust Orthod J 2008; 24: 110-115.

7) Ahrari F, Heravi F, Fekrazad R, Farzanegan F, Nakhaei S. Does ultra-pulse CO2 laser reduce the risk of enamel damage during debonding of ceramic brackets? Lasers Med Sci 2012; 27: 567-574.

8) Ryf S, Flury S, Palaniappan S, Lussi A, van Meerbeek B, Zimmerli B. Enamel loss and adhesive remnants following bracket removal and various clean-up procedures in vitro. Eur J Orthod 2012; 34: 25-32.

9) Abou-Rass M. Crack lines: the precursors of tooth fractures - their diagnosis and treatment. Quintessence Int Dent Dig 1983; 14: 437-447.

10) $\mathrm{Xu} H \mathrm{HH}$, Kelly JR, Jahanmir S, Thompson VP, Rekow ED. Enamel subsurface damage due to tooth preparation with diamonds. J Dent Res 1997; 76: 1698-1706.

11) Habibi M, Nik TH, Hooshmand T. Comparison of debonding characteristics of metal and ceramic orthodontic brackets to enamel: an in-vitro study. Am J Orthod Dentofacial Orthop 2007; 132: 675-679.

12) Dumbryte I, Linkeviciene L, Malinauskas M, Linkevicius T, Peciuliene V, Tikuisis K. Evaluation of enamel micro-cracks characteristics after removal of metal brackets in adult patients. Eur J Orthod 2013; 35: 317-322.

13) Bajaj D, Park S, Quinn GD, Arola D. Fracture processes and mechanisms of crack growth resistance in human enamel. Biol Biomed Mater 2010; 62: 76-82.

14) Dental materials - guidance on testing of adhesion to tooth structure. 2nd ed. ISO TS 11405. Geneva: International Organization for Standardization; 2003.

15) Zachrisson BU, Skogan O, Hoymyhr S. Enamel cracks in debonded, debanded and orthodontically untreated teeth. Am J Orthod 1980; 77: 307-319.

16) Hosein I, Sherriff M, Ireland AJ. Enamel loss during bonding, debonding, and cleanup with use of a self-etching primer. Am J Orthod Dentofacial Orthop 2004; 126: 717-724.

17) Theus M, Urbanek S. Interactive Graphics for Data Analysis. Principles and Examples. FL: CRC Press, Taylor \& Francis Group, 2008. 
18) Schuler FS, van Waes H. SEM-evaluation of enamel surfaces after removal of fixed orthodontic appliances. Am J Dent 2003; 16: 390-394.

19) Park S, Wang DH, Zhang D, Romberg E, Arola D. Mechanical properties of human enamel as a function of age and location in the tooth. J Mater Sci Mater Med 2008; 19: 2317-2324.

20) Zhang YR, Du W, Zhou XD, Yu HY. Review of research on the mechanical properties of the human tooth. Int J Oral Sci 2014; 6: 61-69.
21) Clark DJ, Sheets CG, Paquette JM. Definitive diagnosis of early enamel and dentin cracks based on microscopic evaluation. J Esthet Restor Dent 2003; 15: 391-401.

22) Alessandri Bonetti G, Zanarini M, Incerti Parenti S, Lattuca M, Marchionni S, Gatto MR. Evaluation of enamel surfaces after bracket debonding: an in-vivo study with scanning electron microscopy. Am J Orthod Dentofacial Orthop 2011; 140: 696-702. 